FORMATION Formation emploi

Revue française de sciences sociales

122 | Avril-Juin 2013

Relever les défis de la Validation des Acquis de

l'Expérience (VAE)

\title{
Politique et management de la VAE dans le travail social : le diplôme d'État d'éducateur spécialisé et le dispositif de branche
}

Policy and Management of Accreditation of Prior Learning in the Social Sector: Accessing The State-owned Diploma "Educateur Spécialisé"

Politik und Management der VAE im Bereich Sozialarbeit : Das „Diplôme d'Etat d'éducateur spécialisé "und das Instrument einer Branche

Política y gestión de la VAE en Trabajo social. El diploma oficial de educador especializado y el dispositivo de rama

\section{Pascale de Rozario}

\section{OpenEdition \\ Journals}

Édition électronique

URL : http://journals.openedition.org/formationemploi/3994

DOI : 10.4000/formationemploi.3994

ISSN : 2107-0946

Éditeur

La Documentation française

Édition imprimée

Date de publication : 30 juin 2013

Pagination : 87-114

ISSN : 0759-6340

Référence électronique

Pascale de Rozario, « Politique et management de la VAE dans le travail social : le diplôme d'État d'éducateur spécialisé et le dispositif de branche », Formation emploi [En ligne], 122 | Avril-Juin 2013, mis en ligne le 17 juillet 2013, consulté le 30 octobre 2020. URL : http://journals.openedition.org/ formationemploi/3994; DOI : https://doi.org/10.4000/formationemploi.3994 


\title{
Politique et management de la VAE dans le travail social
}

\section{Le diplôme d'état d'éducateur spécialisé et le dispositif de branche}

\author{
PAscale de Rozario \\ Sociologue \\ Umr - Unité mixte de recherche/Cnrs 3320 Lise - Laboratoire interdisciplinaire pour la \\ sociologie économique/Cnam - Conservatoire national des arts et métiers
}

Résumé

\begin{abstract}
Politique et management de la VAE dans le travail social : le diplôme d'Etat d'éducateur spécialisé et le dispositif de branche

L'article présente d'abord la politique de VAE (validation des acquis de l'expérience) de la branche du travail social, amorcée en 2004 à partir du diplôme d'État d'éducateur spécialisé. Il s'agit d'une politique d'une ampleur inédite en France en matière d'éducation spécialisée. Nous proposons ensuite un angle d'analyse de la VAE original relatif à l'exercice appliqué de ce droit individuel à l'échelle collective du secteur, soit le poids de l'encadrement régional de la VAE par les choix d'orientation des doctrines de recevabilité des demandes administratives d'une part, et les consignes de validation transmises aux jurys, d'autre part. Ces stratégies de gestion confient des mandats très différents à la VAE, droit plurivoque qui divise les acteurs sur les conceptions légitimes de la reconnaissance de l'expérience, nommées ici cultures de la VAE.
\end{abstract}

Mots clés : validation des acquis, action sociale, éducateur spécialisé, approche locale

Abstract

Policy and Management of Accreditation of Prior Learning (APL) in the Social Sector: Accessing The State-owned Diploma "Educateur Spécialisé"

This contribution first portrays the sectorial national policy launched in 2004 by the social partners of the social work sector to reward highly experienced. This policy has no other equivalent in France in terms of scope and targeting. The second part suggests a new original analysis of APL through (a) the more or less restricted degrees of selection applied to single administrative demands by regional management styles of APL and (b) the influence of instructions conveyed to members of jury on their own judgement and expectations regarding the candidates' performance. In effect, these various strategies revealed very different mandates and expectations put on APL, defined as a "polyvocal" right par- 
ties highly differ on, in particular about what would be a fair reward of prior learnings. These conceptions are herewith described as "cultures of recognition".

Key words: validation of acquired skills, help support, special education teacher, local approach

Journal of Economic Literature: I 29

Traduction : Auteur.

La politique de VAE (validation des acquis de l'expérience) de la branche professionnelle associative du travail sanitaire et social, amorcée en 2004, est d'une ampleur inédite. Nous proposons ici un angle original d'analyse de la VAE en interrogeant comment la gestion régionale de la VAE oriente la sélection des candidats lors de leur demande administrative avant leur passage devant les jurys, et pèse sur les résultats au travers des consignes de validation transmises aux membres de jury. Les stratégies de gestion régionales apparaissent très différentes. La VAE, droit plurivoque, divise les acteurs sur les conceptions légitimes de la reconnaissance de l'expérience, nommées ici cultures de la VAE.

Décréter un droit ne suffit pas à le faire appliquer de manière univoque, ni uniforme dans l'ensemble des régions. Nous verrons que le terme de "gouvernance " (Le Galès, 1994) se justifie pour qualifier la VAE, plus encore dans le secteur du travail social où cinq ministères gèrent 143 diplômes. Polycentricité des lieux de décision, multiplicité des décideurs et des niveaux d'intervention, pluralité des références et des procédures caractérisent cette gouvernance nationale ordinaire. S'y ajoute une gouvernance régionale, angle d'observation privilégié de nos travaux de recherche. Ce contexte complexe pose des défis d'actions et de légitimité, mais n’a pas freiné la branche professionnelle des établissements privés à but non lucratif (principalement associatifs) dans sa politique de qualification par la VAE d'environ 28000 professionnels expérimentés, faisant fonction d'éducateurs spécialisés. Quels enjeux ont conduit les partenaires sociaux, les écoles du travail social et les certificateurs à déployer une telle politique de diplômation massive par la VAE ? Comment caractériser ce premier prototype de dispositif d'accompagnement à la VAE intitulé le « DSB VAE DEES " (dispositif de soutien de branche à la VAE pour l'accès au diplôme d'Etat d'éducateur spécialisé) ? Question récurrente : l'accompagnement VAE des candidats a-t-il été plus efficace que leur sélection à l'entrée de ce dispositif? Quels facteurs peuvent expliquer les différences régionales de gestion de la VAE et la variabilité des résultats finaux ? Peut-on parler de diverses conceptions et de cultures de la reconnaissance ? Et si oui, sur quelles convictions reposent-elles?

La première partie de cet article présente les enjeux et le dispositif de cette politique sectorielle dans un contexte de gouvernance des diplômes du travail social. À l'ouverture du diplôme d'État d'éducateur spécialisé à la VAE, 79 écoles du travail social, regroupées en 22 pôles ressources régionaux, sont labellisées et prêtes à déployer ce dispositif. Entre 2005 
et 2006, 1767 salariés sont accompagnés dans toutes les régions et 722 se présentent aux jurys, soit $15 \%$ du total des candidats à la VAE en France (4 880). Dans la deuxième partie, nous présenterons les résultats de validation à partir de l'analyse du parcours de 722 candidats suivis de manière longitudinale depuis les bases de données statistiques d'Unifaf (Fonds d'assurance formation de la branche sanitaire, sociale et médico-sociale à but non lucratif) et d'une évaluation du déploiement de ce dispositif dans neuf régions (406 entretiens qualitatifs auprès du système d'acteurs représentatifs). La recherche, conduite sur deux ans (2005-2007), a permis d'identifier deux facteurs majeurs d'influence des résultats de validation observés en région. Le premier est lié au pilotage et à la gestion régionale de la VAE, le second à différentes cultures de la reconnaissance en usage dans le travail social. Nous constatons que les acteurs régionaux chargés de la VAE la pilotent en s'entendant, en se construisant des doctrines de recevabilité des candidatures, plus ou moins sélectives, et en orientant les critères de jugement des jurys par des initiatives qualifiées de "professionnalisation ". Ainsi, l'analyse de plus de 130 dilemmes de jugements détaillés par une soixantaine de membres de jury interrogés dévoile trois types d'attentes normatives par rapport aux candidats, que l'on peut associer à trois cultures distinctes de reconnaissance de l'expérience. Les jugements de validation oscillent ainsi entre trois options possibles : reconnaître un candidat impliqué et motivé même s'il ne maîtrise pas tous les critères du référentiel du diplôme d'éducateur spécialisé, selon une conception " promotionnelle » de la VAE ? Reconnaître la qualité d'un exercice d'évaluation qui fait coïncider l'expérience et au référentiel de ce diplôme (conception « docimologique») ? Ou dans la singularité de l'expérience individuelle, doit-on plutôt reconnaître des apprentissages transférables dans d'autres contextes d'activités, ce que devrait garantir le diplôme d'État dans une conception « transversale " et universaliste de la VAE ?

\section{Les enjeux sectoriels d'un dispositif de VAE}

Trois principaux motifs sont invoqués par la CPNE (Commission paritaire nationale de l'emploi) du travail social lorsqu'elle entérine les travaux de la branche professionnelle et d'Unifaf (délibération CNPE du 14 janvier 2003) et appuie la politique sectorielle de VAE :

- la nécessité de normaliser la situation d'environ 28000 professionnels faisant fonction d'éducateurs spécialisés dans un contexte de réformes et de décentralisation ;

- l'anticipation des départs en retraite annoncés par le Contrat d'études prospectives du secteur (Credoc, 2002) et la perception partagée d'un besoin de professionnalisation ou de renouvellement professionnel (Pinaud, 2004);

- la facilitation de l'accès aux diplômes de l'éducation spécialisée par la VAE. 
Nous verrons que le dispositif de VAE Educateur spécialisé survient dans un contexte spécifique caractérisé par la décentralisation des formations du travail social en région, l'identification d'un nombre significatif de professionnels expérimentés sans diplôme, une génération professionnelle vieillissante à renouveler et la nécessité de réécrire les référentiels des diplômes du travail social afin de permettre leur accès à des personnes expérimentées via la VAE. Il convient de mentionner par ailleurs un contexte plus large de " mutations " du travail social à la fois internes et externes, notamment par l'introduction d'un rapport différent entre usagers et professionnels du travail social, plus basé sur une égalité de droit avec un questionnement profond du rapport « expertusager " des politiques sociales (voir Salmon, 2010 ; Capul, 2007 ; Janvier, 2004 ; Chopart, 2000, entre autres).

\subsection{Un contexte de rationalisation et de réformes}

En plus d'illustrer la partition historique de ce secteur entre le domaine sanitaire et le domaine social (Jaeger, 2006 ; Conseil supérieur du travail social, 2007), les données de l'Observatoire de branche permettent de situer les professionnels ciblés. Ce secteur en forte croissance emploie plus de 550000 salariés et 15726 structures. Le tableau 1 liste les dix types d'organisations les plus représentatives du secteur (Enquête emploi Unifaf, 2007). Le domaine sanitaire et médico-social représente $35 \%$ des emplois principalement occupés par les aides-soignants $(35 \%)$, les infirmiers $(33 \%)$ et les agents de service hospitaliers (22\%). Le domaine social regroupe l'équivalent d'emplois $(34 \%)$ et trois principaux métiers : les éducateurs spécialisés $(27 \%)$, les assistants médico-psychologiques (19\%) et les moniteurs éducateurs (14\%). Le troisième domaine concerne les métiers administratifs et de gestion (tableau 2).

La présence de faisant-fonction s'explique par l'organisation et l'histoire spécifiques de l'éducation spécialisée et des structures associatives ; toutefois, leur existence semble poser problème dans le contexte de réformes et de rationalisation notamment porté par la loi 2002-2 "rénovant l'action sociale et médico-sociale et la loi 2005-102 "pour l'égalité des chances, la participation et la citoyenneté des personnes handicapées ». S'ajoute la décentralisation des formations sanitaires et sociales au niveau des conseils régionaux via la loi du 13 août 2004 "relative aux libertés et responsabilités locales". 
Effectif des 10 principales catégories d'établissements (Enquête emploi 2007, Unifaf, 2008, p.9)

\begin{tabular}{|l|c|c|}
\hline \multicolumn{1}{|c|}{ Type d'organisation } & $\begin{array}{c}\text { Effectifs (nbre } \\
\text { de salariés) }\end{array}$ & Effectifs (en \%) \\
\hline Etablissements hospitalièrs en court séjour & 64600 & $8 \%$ \\
\hline Instituts médico-éducatifs et médico-psychologiques (IME+IMP) & 47100 & \\
\hline Maison de retraite & 44200 & \\
\hline Maison d'enfants à caractère social (MECS) & 30600 & $5 \%$ \\
\hline Centre d'aide par le travail (CAT/ESAT) & 27200 & $5 \%$ \\
\hline Foyer d'hébergement pour adultes handicapés & 27000 & $5 \%$ \\
\hline Foyer de vie pour adultes handicapés & 23000 & $4 \%$ \\
\hline Centre hospitalier de lutte contre les maladies mentales & 17600 & $3 \%$ \\
\hline Maison d'accueil spécialisé (MAS) & 16300 & $3 \%$ \\
\hline Administration/Siège & 16000 & $3 \%$ \\
\hline
\end{tabular}

Tableau 1

Répartition des emplois par domaines (Enquête emploi 2007, Unifaf, 2008, p. 13)

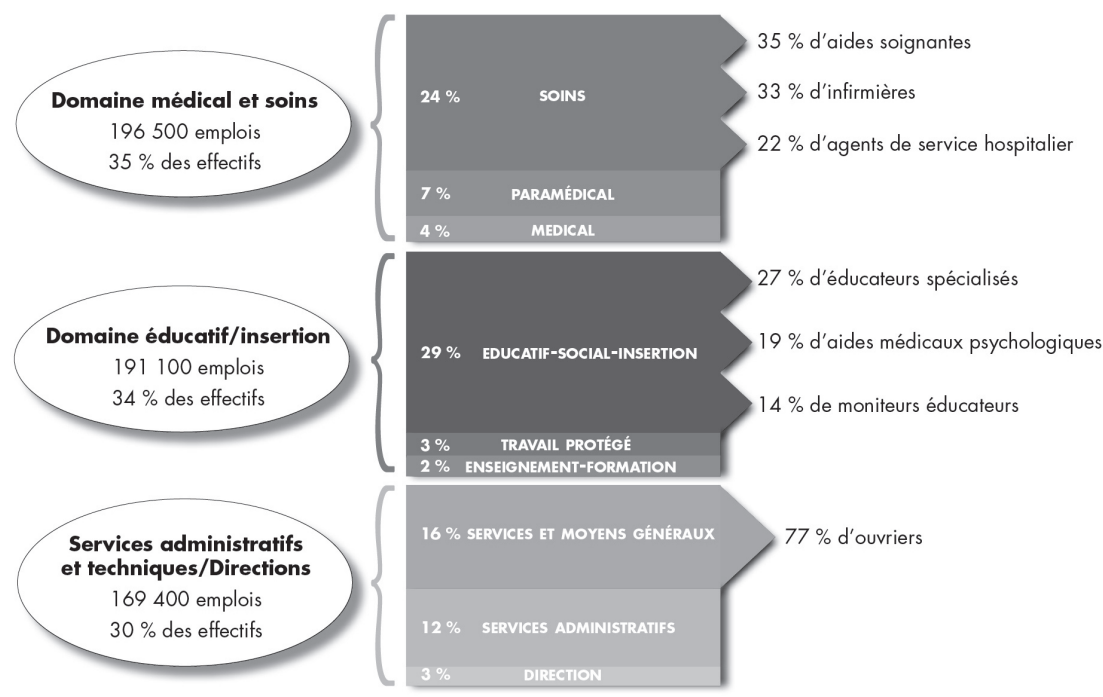

Tableau 2

Note de lecture : Dans le tableau 1, les « établissements hospitaliers en court séjour » sont le type d'organisation le plus présent dans le secteur du travail social en 2007.

Dans le tableau 2, le « domaine médical et soins » représente 196500 emplois soit $35 \%$ des emplois du secteur. La composante « soin » de ce domaine représente $24 \%$ du secteur, en soin, les aides-soignants constituent $35 \%$ des emplois ( $35 \%$ parmi les $24 \%$ ) (*).

Sigles : Unifaf « Fonds d'assurance formation des entreprises de la branche sanitaire, médico-sociale et sociale à but non lucratif ».

$\left({ }^{*}\right)$ : La comparaison des données d'emploi entre 2008 et 2012 démontre trois choses:

- I'attractivité continue des métiers concernés par la VAE avec une croissance totale d'emploi de $26 \%$ (21 \% pour les éducateurs spécialisés ; $18 \%$ pour les assistants médico-psychologiques et $26 \%$ pour les moniteurs éducateurs). Ces métiers font aussi parti du « Top 5 » des emplois référents cités par l'enquête emploi publiée en 2012 ; 
- la croissance du nombre des établissements (+ $25 \%$, le nombre d'établissements total en 2012 est de 20 000), notamment dans le secteur du handicap et du vieillissement;

- l'impact positif de la VAE et du dispositif de branche généralisé (DSB VAE) puisqu'en 2012 avec deux indicateurs, le nombre de faisant fonction a été divisé par 2 dans ces métiers et le recrutement devenu plus facile d'éducateurs spécialisés, alors qu'en 2007, 12 \% des établissements interrogés se plaignaient de la difficulté à recruter.

Lien Enquête Emploi L'Observatoire \& Unifaf 2008 : http://www.obs-professionsolidaires.fr/attached_file/componentld/kmelia283/ attachmentld/13255/lang/fr/name/enquete_emploi\%20-020mars\%202008.pdf

\subsection{Augmenter le niveau de qualification et d'encadrement}

Le contrat d'études prospectives du secteur (Credoc, 2002) pointe la faiblesse des effectifs de cadres et les difficultés prévisibles de leur renouvellement lors du départ en retraite de la génération actuellement en poste, dont les éducateurs spécialisés de niveau de qualification III. Des diplômés pour l'encadrement intermédiaire et supérieur feraient défaut. Le rapport sur la prospective des métiers pour 2015 souligne l'attractivité du travail social et plusieurs enjeux de professionnalisation (Chardon et al., 2007), dont une répartition plus équilibrée de la population active aux différents niveaux de qualification possibles, et ce dans un contexte où les actifs et les diplômes de ce secteur sont majoritairement de niveau $\mathrm{V}^{1}$.

L’analyse des données du ministère chargé des Affaires sociales (DGAS, juillet 2007) permet par ailleurs de dresser une cartographie des 143 diplômes de ce secteur (Rapport de Rozario, 2008). Le tableau 3 confirme le poids des diplômés de premiers niveaux de qualification $\mathrm{V}^{2}$ (59 \% du total des diplômés) et de niveau IV ( $25 \%$ du total des diplômés) ; la faiblesse du niveau III (14\% des diplômés) et l'explosion récente des diplômes universitaires d'encadrement (37 licences et 68 masters !). Ainsi, la majorité des diplômes du secteur se situe à un niveau de qualification peu élevé et il convient de parler de co-gestion entre certificateurs plutôt que de politique publique des diplômes.

1. Pour l'anecdote, au cours du colloque européen organisé au CNAM sur les enjeux de la VAE en Europe (De Rozario, 2005) à partir d'une recherche comparative (Projet européen VAP et Entreprises, Programme Leonardo da Vinci, Commission européenne DGV Education et Culture), le représentant ministériel belge (M. Vanraepenbush) avait été stupéfait de la présentation des métiers auprès des enfants, adolescents et famille et n'avait pu s'empêcher de formuler cette critique que personne n'a contredite (présentation du référentiel par les Maisons familiales rurales) : "Vous en France, vous venez de décrire en long et en large tout ce que vous attendez d'une assistante maternelle ou de ce que vous appelez une aide médico-psychologique. C'est énorme, très long, incroyable. Et vous ne donnez que le niveau $V$ ? Elles ne touchent que ce salaire-là? Elles font le rôle de mère de famille, ce n'est que cela la valeur que vous attribuez à ce rôle si important?"

2. Nomenclature des niveaux de formation 1969: Niveau V : diplômés d'un brevet d'études professionnelles (BEP) (deux ans de scolarité au-delà du premier cycle de l'enseignement du second degré) et du certificat d'aptitude professionnelle (CAP) ou équivalents. Niveau IV : diplômés du baccalauréat, du brevet de technicien (BT) ou équivalents. Niveau III : diplômés d'un brevet de technicien supérieur (BTS), du diplôme universitaire de technologie (DUT) ou équivalents. Niveau II : diplômés de niveau égal ou supérieur à celui de la licence ou équivalents. Niveau I : diplômés de niveau égal ou supérieur à un niveau de master ou équivalents. 
Les mobilités verticales (vers les niveaux d'encadrement) et les mobilités horizontales (entre diplômes de plusieurs ministères) restent difficiles à mettre en ouvre.

Tableau 3

\section{Cartographie des 143 diplômes du travail social, adapté à partir d'« Orientation pour} les formations sociales 2007-2009 »

\begin{tabular}{|c|c|c|c|c|c|c|c|c|c|c|c|}
\hline \multicolumn{12}{|c|}{5 certificateurs publics * (143 diplômes/94348 diplômés) } \\
\hline \multicolumn{6}{|c|}{$\begin{array}{l}\text { Co-gestion } \\
\text { (6 diplômes) }\end{array}$} & \multicolumn{6}{|c|}{ Mono Gestion (137 diplômes) } \\
\hline Niveaux & Diplômes & $\%$ & Diplômés & $\%$ & AS/EN & JS/AS & EN & AS & Agr. & TE & JS \\
\hline I & 70 & $49 \%$ & 210 & $\begin{array}{c}0,20 \\
\%\end{array}$ & 1 & $-/-$ & 68 & 1 & $-1-$ & $-/-$ & - \\
\hline II & 40 & $28 \%$ & 1000 & $1 \%$ & $-1-$ & $-/-$ & 37 & 2 & $-1-$ & $-1-$ & 1 \\
\hline III & 14 & $10 \%$ & 13781 & $15 \%$ & 3 & 1 & 6 & 2 & 1 & 1 & $\%$ \\
\hline IV & 8 & $6 \%$ & 23380 & $25 \%$ & 1 & $-1-$ & 2 & 1 & 1 & 2 & 1 \\
\hline \multirow[t]{5}{*}{ V } & 11 & $8 \%$ & 55977 & $59 \%$ & $-1-$ & $-1-$ & 4 & 3 & 2 & 2 & - \\
\hline & 143 & $100 \%$ & 94348 & $100 \%$ & 7455 & 342 & 55616 & 15852 & 8981 & 4089 & 2013 \\
\hline & & & $\begin{array}{l}\text { Nombre de } \\
\text { diplômés }\end{array}$ & & 5 & 1 & 117 & 9 & 4 & 5 & 2 \\
\hline & & & \% Total & & 3,5 & 0,7 & 81,8 & 6,3 & 2,8 & 3,5 & 1,4 \\
\hline & & & $\begin{array}{c}\text { \% diplômés/ } \\
\text { Total }\end{array}$ & & 7,9 & 0,4 & 58,9 & 16,8 & 9,5 & 4,3 & 2,1 \\
\hline
\end{tabular}

Note de lecture : Le niveau I de formation dans le travail social est constitué de 70 diplômes (soit $49 \%$ des diplômes du secteur) et de 210 diplômés (soit 0.20 \% des diplômés). À ce niveau de formation, un diplôme est en cogestion entre les ministères des Affaires sociales et Jeunesse \& Sport, et 6 sont en mono-gestion par l'Éducation nationale.

Source : Construit à partir des chiffres DGAS, 2007.

* Certificateurs : AS : Affaires sociales ; EN : Education nationale + sup. ; Agr. : Agriculture ; TE : Emploi JS : Jeunesse et Sports.

L’enjeu de la VAE est double : élever le niveau de qualification des praticiens de niveaux $\mathrm{V}$ et IV, principalement les moniteurs éducateurs et les aides médico-psychologiques faisant fonction d'éducateurs spécialisés, et augmenter le taux d'encadrement intermédiaire de niveau III, dont les éducateurs spécialisés. Le DEES (diplôme d'État d'éducateur spécialisé) est également un diplôme emblématique et très valorisé de l'éducation spécialisée ; s'il se prête à la VAE - c'est l'hypothèse des partenaires sociaux promoteurs du dispositif - la majorité des autres diplômes du travail social qui ne sont pas encore ouverts à la VAE suivront, bien que dans un contexte de réticence vis-à-vis de cette démarche. 


\subsection{Faciliter l'ouverture des diplômes du travail social à la VAE : le chantier des référentiels}

En dehors du cas du DEAVS (diplôme d'État d'auxiliaire de vie sociale de niveau V) ouvert à la VAE en 2003, les référentiels de diplôme du travail social sont alors à créer. Une méthode reste à trouver, ministères, partenaires sociaux et experts qualifiés devant s'entendre au sein de la nouvelle Commission professionnelle consultative du travail et de l'intervention sociale, créée par l'arrêté du 11.09.2002. Les analystes décrivent, avec la généralisation de la VAE à tous les diplômes, la survenue d'un "ordre certificatoire " (Maillard, 2008) où les consultants et spécialistes des référentiels d'activité et de compétences s'emparent du pouvoir technique de redéfinir le métier par le diplôme, puis par le référentiel (Neyrat, 2007 ; de Rozario, 2005). Le travail social n’échappe pas à ce processus. Or, la décision d'ouvrir le diplôme d'État d'éducateur spécialisé et l'écriture des référentiels ad hoc sont problématiques ; en effet, le DEES ${ }^{3}$ dépend de deux ministères : le ministère de l'Éducation nationale, puisque le recteur d'Académie signe le parchemin de ce diplôme, et le ministère des Affaires sociales par son inscription dans le Code de l'action sociale et des familles (CASF). Qui décide ? Qui fait et selon quelle philosophie du métier, du diplôme et du référentiel, les parties prenantes ayant des conceptions sensiblement variables, sinon conflictuelles sur ces questions?

Dans ce contexte, la branche du travail social prend l'initiative - et les certificateurs publics la lui laissent - des travaux d'écriture du nouveau référentiel d'éducateur spécialisé et du dispositif de soutien VAE de branche, qui démarrent dès 2000. Ces travaux sont techniquement confiés à l'Opca Unifaf - dont on soulignera l'expertise d'ingénierie en gestion des ressources humaines - et à quelques experts des écoles du travail social. En 1995, l'Opca avait déjà expérimenté le dispositif VAP 92 (reconnaissance des acquis d'expérience de type professionnels) pour l'accès au diplôme d'État d'aide médico-psychologique (DEAMP) dans six régions, avec l'appui de quelques écoles du travail social convaincues. Notons aussi le rôle particulièrement moteur de la présidence paritaire d'Unifaf, stable depuis vingt ans, entre les représentants syndicaux de salariés et d'employeurs, par ailleurs proches des écoles du travail social. Dans le cas présent, la polysémie de la VAE a servi des alliances sur des conceptions pourtant différentes : les représentants de salariés mettent plutôt en avant une démarche de promotion profes-

3.Les 5 diplômes co-gérés par deux principaux ministères, sachant que les autres peuvent être co-signataires, mais intervenir dans une moindre mesure dans la mise en œuvre de la VAE et la refonte des certifications :

- le DEIS (diplôme d'État d'ingénierie sociale) de niveau I ;

- le DCESF (diplôme de conseiller en économie sociale et familiale) de niveau III ;

- le DEES (diplôme d'État d'éducateur spécialisé) de niveau III ;

- le DEETS (diplôme d'État d'éducateur technique spécialisé) de niveau III ;

- le CAFME (Certificat d'aptitude aux fonctions de moniteur éducateur) de niveau IV. 
sionnelle et sociale garantie par un diplôme d'État ; les représentants d'employeurs une démarche de gestion prévisionnelle des emplois et des compétences (GPEC).

Il aura donc fallu attendre deux ans et un corpus compliqué de quatre textes pour ouvrir le diplôme d'État d'éducateur spécialisé à la VAE : la circulaire 2003-127 du 1/08/03 du ministère de l'Éducation nationale ; l'arrêté du 12 mars 2004 modifiant les critères de sélection et de formation des éducateurs spécialisés pour intégrer la VAE et diffuser le nouveau référentiel de diplôme ; la circulaire des Affaires sociales DGAS/4A/2004/333 du 7 juillet 2004 reprise par celle du ministère de l'Éducation nationale (2004-132) pour l'organisation concrète de la VAE au sein de chaque réseau. Ainsi, faire VAE ne se décrète pas avec effet immédiat, loin s'en faut.

\subsection{Le soutien à la VAE Educateur spécialisé : un dispositif original et expérimental}

Les partenaires sociaux et l'Opca Unifaf ont conçu le dispositif de soutien de branche dans l'optique de maîtriser le plus de paramètres possibles. Soucieux des résultats de validation, ses concepteurs introduisent une sélection des candidats (des pré-requis d'accès au dispositif et « l'hypothèse de bonne fin » dans les étapes 1 et 2) et des actions d'accompagnement VAE (" l'appui méthodologique »VAE, des " mises en situation professionnelle » et des "séquences formatives " dans les étapes 3 et 4). Le schéma 1 détaille ce dispositif particulièrement riche d'une durée maximale de 190 heures, dont 175 heures financées dans le cadre du plan de formation. Il présente le dispositif expérimental mis en place dès 2004 et le nouveau dispositif instauré à partir de 2008, simplifié et étendu à huit autres diplômes. Notons que le « livret 1 " est constitué des documents administratifs qui permettent d'accepter une demande de VAE (nombre d'années d'expérience suffisantes, expérience en lien avec le diplôme visé et suffisante par rapport à la demande de VAE) et le " livret 2 » du document d'auto-évaluation et autres preuves associées, examinés par les membres de jury pour décider l'octroi de la totalité du diplôme, d'une partie ou d'un rejet de la demande (en ce cas, aucune partie du diplôme n'est validée).

En matière de sélection des candidats, la branche n'innove pas. Les quinze monographies d'entreprises montrent que tous les dispositifs collectifs décrits sélectionnent les candidats les plus proches de la validation (CEE, 2008). L'hypothèse de bonne fin s'apparente aux " études de faisabilité » ou aux " diagnostics » décrits, conduits par les organismes de formation de l'Éducation nationale (Greta - groupement d'établissements publics locaux d'enseignement). Le rapport Dubouchet (2005) et nos enquêtes convergent : ces pratiques ont également cours au sein de quelques cellules régionales d'information et de conseil en VAE ou équivalents, c'est-à-dire au moment d'informer les candidats à la VAE. En quelques heures, il s'agit de vérifier les chances de validation du candidat en rapprochant les expériences sélectionnées des différents domaines ou fonctions du référentiel de métier du diplôme visé. Cette phase d'information et de conseil définie par le législateur comme 
gratuite est centrale en matière d'évaluation de la faisabilité d'une demande et d'orientation vers un diplôme correspondant le mieux à l'expérience.

Les candidats accèdent au dispositif de soutien de branche s'ils possèdent un diplôme de niveau de qualification inférieur au DEES (V et IV) dans la filière éducative, ce qui cible les diplômes de moniteur éducateur (IV) et d'aide médico-psychologique (V), une expérience plus longue de cinq ans pour les aide médico-psychologiques ou un diplôme universitaire supérieur, et la chance de valider au moins trois des quatre fonctions du référentiel d'éducateur spécialisé. Après évaluation (2006-2008), ces conditions de sélection seront supprimées pour la deuxième version du DSB ; en effet, elles contribuent finalement peu à la réussite des candidats et surchargent inutilement les pratiques de sélection régionale déjà en ouvre au niveau des dispositifs d'information-conseil en VAE ou des autres opérateurs d'accompagnement VAE.

\section{Schéma 1}

Représentation du dispositif de soutien de la branche professionnelle du travail social privé à but non lucratif à la VAE pour le diplôme d'État d'éducateur spécialisé

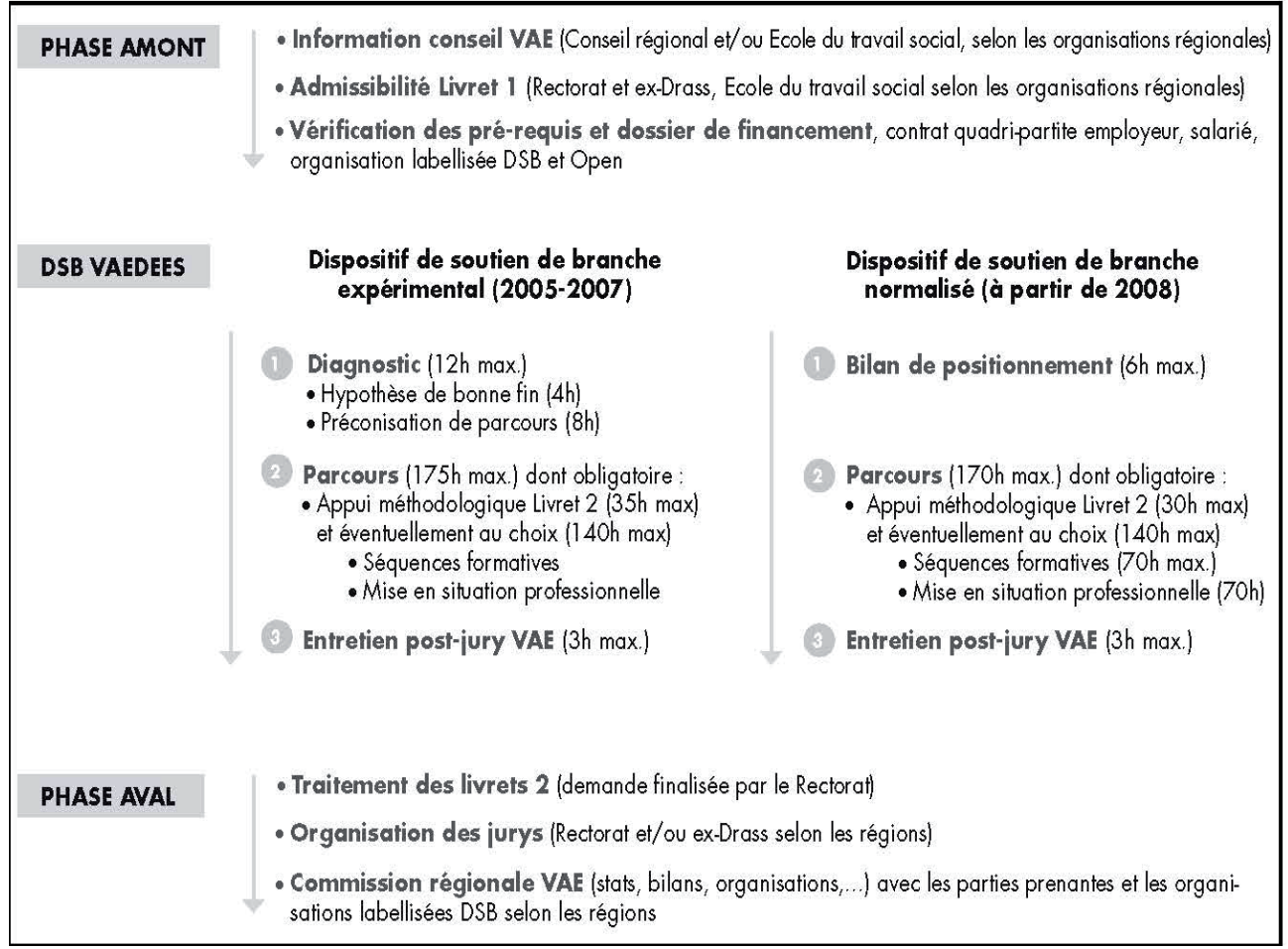




\section{Encadré 1}

\section{Les 4 fonctions du nouveau référentiel d'éducateur spécialisé devenus par la suite « domaines de compétences »}

F1 «Etablissement d'une relation, diagnostic éducatif »;

$\mathrm{F} 2$ «Accompagnement éducatif de la personne ou du groupe»;

F3 « Conception et conduite d'une action socio-éducative au sein d'une équipe » ;

F4 «Construction d'un cadre d'analyse et d'une expertise des pratiques éducatives et sociales ».

Le parcours d'accompagnement est original car il associe à l'accompagnement méthodologique classique en VAE, appelé " l'appui à la démarche ", des " séquences formatives " et des "mises en situation professionnelle ", sachant que ni les stages professionnels ni les actions de formation ne sont considérés comme des actions de VAE (l'action de VAE consiste à formaliser des savoirs acquis d'expérience et les rapprocher des savoirs acquis par une formation. Cet exercice spécifique ne peut ni se réaliser par la formation, ni s'acquérir par une expérience professionnelle). Juridiquement, il convenait de distinguer leurs particularités.

L'analyse statistique du descriptif de 3045 actions financées rapportées aux 1691 candidats retenus dans ce dispositif permet d'identifier un parcours type d'accompagnement d'une durée moyenne de 89 heures et des mises en situation professionnelle ne concernant que $2 \%$ des candidats (Rapport de Rozario, 2008). L'appui à la démarche de VAE se centre sur l'auto-évaluation et la présentation des situations choisies dans la structure du livret 2 et la préparation de l'entretien oral (principalement sa dédramatisation). Les "séquences formatives" portent soit sur l'appropriation du nouveau référentiel d'éducateur spécialisé et son vocabulaire, peu familier aux professionnels, soit sur la réappropriation des réformes du travail social. Les « mises en situation professionnelle " sont utilisées pour vérifier le projet de VAE et de mobilité et déclencher une posture réflexive d'auto-évaluation, notamment pour les candidats dont le cadre professionnel et le public ont très peu varié dans le temps. C'est le cas d'un candidat resté plus de dix ans dans une structure spécialisée auprès de personnes âgées, par exemple, qui n’a expérimenté que la psychologie, le comportement et la relation avec ce type de public. Il arrive que de nombreux candidats rêvent alors de travailler dans l'éducation spécialisée, les milieux ouverts auprès des enfants et adolescents mais sans véritablement savoir si les interactions avec ce nouveau public lui conviendront. Les mises en situation professionnelle, courtes, ont principalement permis de vérifier la pertinence de ce désir de changement de public et de le concrétiser sous forme du projet de VAE. 


\section{Encadré 2}

\section{Précisions méthodologiques (Evaluation de la politique de branche et recherche conduites de 2006 à 2007)}

Dispositif de pilotage (24 personnes) : GPN (groupe de pilotage national dont CPNE - commission paritaire nationale de l'emploi) GT (groupe technique Unifaf et écoles du travail social), GP (groupe projet, Unifaf), Chercheurs (P. de Rozario, Umr Cnrs 3320 Lise-Cnam (Laboratoire interdisciplinaire de la sociologie du conservatoire national des arts et métiers) et enquêteurs (C. Delors et H. Tran, Cnam)

- Construction d'une base de données spécifique (170 variables pour 1767 salariés candidats à la VAE) :

Bases de données employeurs et salariés du logiciel Sirius d'Unifaf $X$ Base de données des actions DSB (3045 descriptifs) X Résultats régionaux de validation (2005 et 2006, source : ministère de l'Education nationale, MEN).

- Phases de la recherche

Phase 1 - Construction du cadre théorique multi-niveaux.

Phase 2 - Construction de la base de données de recherche à partir de deux bases de données. Construction des typologies.

Phase 3 - Echantillonnages (régions et acteurs) - Test du dispositif d'enquête avec la région Midi-Pyrénées.

Phase 5 - Enquêtes dans les 8 autres régions choisies.

Phase 6 - Analyses et traitement - Rapport final (338 p.).

Phase 7 - Restitution des résultats ( 9 colloques régionaux) et colloque final au Cnam (350 personnes - 11/12/2009).

- Enquêtes régionales (9 Régions) : lle-de France, Rhône-Alpes, Aquitaine, Bretagne, Midi-Pyrénées, Alsace, Centre, Bourgogne et Picardie (choix en fonction de la typologie de complexité de coordination assumés par les 28 écoles du travail social labellisées "pôle ressource VAE »).

- Entretiens conduits (406) : identification des dilemmes professionnels, des zones d'incertitude et de trois types de ressources mobilisées (les règles du jeu, la communication et les conceptions de la reconnaissance).

- Typologie du système d'acteurs de la VAE DEES (diplôme d'État d'éducateur spécialisé) :

- Employeurs selon leur usage moyen du dispositif par région (grand utilisateur, moyen utilisateur, petit utilisateur) - 62 entretiens ;

- Salariés selon le parcours et les résultats de validation (non sélectionnés dans le DSB (dispositif de branche), abandon, validation totale, partielle, aucune validation, en cours de parcours) - 116 entretiens ;

- Les 28 pôles ressources VAE labellisés, par degré de complexité de coordination : nombre d'écoles du travail social $X$ nombre de candidats suivis (coordination complexe, simple et bilatérale)- 111 entretiens (acteurs de l'Opca (organisme paritaire collecteur agréé) et des écoles du travail social) ;

- Acteurs régionaux impliqués (ex-Drass - direction régionale des Affaires sanitaires et sociales), Education nationale : Rectorat et Dava - Dispositif académique de validation des acquis) - Conseil régional : responsable VAE et cellules d'information-conseil) - 55 entretiens ; 
- Membres de jury par statut (ex-Drass, Education nationale, employeurs et salariés, formateurs, Jeunesse et Sport, Justice) - 64 entretiens (dont acteurs précédents) ;

- Réalisation de 15 entretiens filmés;

- Concepteurs du dispositif - 27.

Considéré comme expérimental, tant par son ampleur que par la complexité de son ingénierie, ce dispositif a fait l'objet d'une évaluation complémentaire à ces analyses statistiques, assortie d'un dispositif de recherche dont les caractéristiques figurent dans l'encadré qui suit. Le présent article ne présente qu'une synthèse et une partie de la masse d'informations recueillies et d'analyses statistiques et qualitatives produites, qui feront l'objet de publications ultérieures.

\section{La validation : quand management régional et cultures de la reconnaissance se combinent}

Les résultats de validation des 1767 salariés inscrits dans ce dispositif de VAE sont plutôt satisfaisants puisque sur 722 candidats accompagnés présents aux jurys en 2005 et 2006, $41 \%$ ont obtenu la totalité du diplôme d'État d'éducateur spécialisé grâce à leur expérience ; $39 \%$ ont partiellement validé le diplôme et $20 \%$ n’ont rien validé. Les résultats de validation totale et de validation partielle sont significativement plus importants que ceux des candidats qui ont suivi d'autres voies d'accompagnement. L'analyse plus fine des données statistiques de parcours des 1767 et des résultats de validation a permis de détecter des modèles de validation très différents selon les régions ; c'est une des originalités de cette recherche mise en exergue dans cet article. Dans certaines régions classées dans une catégorie de résultats de VAE favorables, on observe, sur plusieurs années, une tendance systématique à plus de validations totales que le cumul des validations partielles et des "non-validation " et significativement moins de non-validation (voir les deux combinaisons favorables du tableau 5). Quelques régions donnent à voir des résultats systématiquement moins favorables : significativement peu de validations totales et un taux de non-validations plus élevé que les autres régions (voir les combinaisons défavorables du tableau 5). Ici, les entretiens qualitatifs avec les acteurs régionaux chargés du pilotage de la VAE, les candidats, les employeurs et les membres de jury interrogés sur les dilemmes de validation rencontrés se sont avérés centraux pour expliquer ces tendances régionales relativement stables d'une année sur l'autre. Nous montrerons, dans cette deuxième partie, d'une part, l'existence de doctrines régionales de management de la VAE, plus ou moins sélectives, et qui orientent la sélection des candidats lors de leur demande administrative bien avant leur passage devant les jurys ; d'autre part, nous mettrons en exergue, au niveau des jurys, des initiatives dites de "professionnalisation " dont 
l'influence est certaine sur les jugements de validation. Trois formes de délibération correspondant à différentes cultures de la reconnaissance professionnelle seront détaillées quant aux attentes de comportements des candidats à la VAE. Ces pratiques parfois informelles, dont l'objectif énoncé est une meilleure " coordination régionale » de la VAE, constituent un frein à l'exercice du droit individuel à la reconnaissance de l'expérience, où priment des considérations managériales, sinon managérialistes, relativement taboues (Chanlat, 2007).

Sans surprise, les candidats sont majoritairement des aides médico-psychologiques, des moniteurs éducateurs et des éducateurs de jeunes enfants (diplômes du secteur social de niveau de qualification inférieure et en lien avec le diplôme d'État d'éducateur spécialisé). Ils ont une moyenne d'âge de 41 ans et de 11 ans d'expérience professionnelle dans le travail social. $30 \%$ sont des hommes et $70 \%$ des femmes, correspondant à la population générale du secteur selon le CEP (2002). Les candidats proviennent de trois domaines d'activités décrits par le Contrat d'études prospectives du travail social (Credoc, 2002) : $60 \%$ du secteur du handicap (catégories CEP 1 et 2 dans le Contrat d'études prospectives cité), $22 \%$ de celui des familles, adultes et enfants en difficulté (CEP 4 et 5 ) et $6 \%$ du secteur sanitaire (hors CEP). Ces candidatures hors secteur signalent des besoins en éducation spécialisée dans les établissements de soins en psychiatrie, en toxicomanie et les centres de rééducation fonctionnelle.

Un tel dispositif devait également démontrer sa performance en termes de résultats de validation, dans un contexte où le législateur a considéré que les candidats pouvaient parfaitement réussir en candidature libre, c'est-à-dire sans appui spécifique. Le tableau 4 illustre que les 722 candidats suivis par ce dispositif ont effectivement obtenu de meilleurs résultats de validation que les autres candidats (plus de validations totales et moins d'absences de validation), en 2005 et 2006. Ce constat conduira d'ailleurs à reconduire ce dispositif après simplification pour sept autres diplômes ${ }^{4}$. Le tableau 4 montre que $57 \%$ des candidats suivis par le dispositif de branche valident l'intégralité du diplôme d'État d'éducateur spécialisé contre $41 \%$ pour les autres candidats. Le taux de validation totale est donc significativement plus élevé via ce dispositif. En revanche, le taux de validation partielle, respectivement de $34 \%$ pour les candidats DSB et de $39 \%$ pour les autres candidats, est relativement proche. Enfin, les candidats ayant suivi le DSB ne sont que $9 \%$ à ne rien valider contre $20 \%$ de candidats hors dispositif. Il semble donc que ce dispositif limite les non-validations, soit les cas où les acquis d'expérience ne coïncident pas, selon les jurys, aux attentes du diplôme.

4. Diplôme d'Etat (DE) moniteur éducateur, DE technicien d'intervention sociale et familiale ; DE éducateur de jeunes enfants; DE éducateur technique spécialisé ; DE assistant de service social ; DE médiateur familial et Certificat d'aptitude aux fonctions d'encadrement et de responsable d'unité d'intervention sociale. 


\section{Tableau 4}

Comparaison des résultats de validation entre candidats DSB (dispositif de branche) et autres candidats au diplôme d'État d'éducateur spécialisé

\begin{tabular}{|c|c|c|c|c|c|}
\hline & 2005-2006 & $\%$ & Dont DSB & $\begin{array}{c}\text { \% } \\
\text { du total des } \\
\text { validations }\end{array}$ & $\begin{array}{c}\% \text { des validations de la } \\
\text { population DSB }\end{array}$ \\
\hline Validations totales & 1994 & $41 \%$ & 415 & $21 \%$ & $57 \%$ \\
\hline Validations partielles & 1887 & $39 \%$ & 243 & $13 \%$ & $34 \%$ \\
\hline \multirow[t]{2}{*}{ Non-validations } & 999 & $20 \%$ & 64 & $6 \%$ & $9 \%$ \\
\hline & 4880 & $100 \%$ & 722 & $-1-$ & \\
\hline
\end{tabular}

Source : Rapport de Rozario, 2008.

Nous avons observé que ces moyennes nationales ne représentaient pas certaines combinaisons de validation régionales, apparemment stables d'une année sur l'autre. Les candidats et des employeurs interviewés mentionnaient par ailleurs leur choix de se présenter aux jurys d'une autre région que la leur avec la conviction que leurs jurys régionaux étaient " contre la VAE ». Les entretiens avec les acteurs régionaux révélaient également des conceptions et des visions de la VAE distinctes, sinon opposées. Certains ne prenaient en considération - et ce dès l'information et le conseil aux candidats - que l'expérience professionnelle alors que la loi de modernisation reconnaît d'autres expériences hors emploi. D'autres jugeaient qu'un praticien n'ayant travaillé qu'auprès de personnes handicapées ne serait pas capable d'exercer auprès d'autres publics, le cantonnant ainsi dans son activité d'origine et une hyper spécialisation fonctionnelle. L'analyse comparée des combinaisons de validation régionales a permis de construire des modèles de validation avec deux orientations : une combinaison de résultats plutôt favorables et une combinaison de résultats plutôt défavorables. L'accompagnement, le financement de la démarche de VAE et la qualité du candidat par rapport à l'exercice ne sont donc pas les seuls facteurs explicatifs des résultats de VAE, contrairement à ce qui est habituellement dit de la VAE. L'analyse de la répartition du taux de validations totales (VT), partielles (VP) et nulles (NV) par rapport aux moyennes nationales a permis d'identifier quatre configurations de validation différenciant nettement les régions.

Deux configurations sont jugées positives en termes de résultats : des validations totales significativement supérieures à la moyenne nationale avec des validations nulles inférieures au taux national de $9 \%$ (combinaison de validation [VT > VP + NV]) et l'absence de validation nulle sur deux ans (combinaison de validation [O NV]). $64 \%$ des candidats accompagnés par le dispositif de branche se situent dans ce premier type de configuration contre $11 \%$ des autres candidats. Deux configurations sont estimées défavorables et surtout difficiles à gérer dans le suivi des candidats après leur passage devant les jurys : des validations partielles et nulles supérieures aux taux nationaux et peu de validations totales (combinaison de validation [VP+NV > VT]) et des taux de validations nulles supérieurs 
à la moyenne nationale $([\mathrm{NV}++]) .89 \%$ des candidats au DEES, en 2005 et 2006, présentent des résultats de validation défavorables, tous dispositifs confondus. Parmi eux, $36 \%$ sont des candidats accompagnés par le dispositif de branche. Ces analyses ont permis d'affirmer qu'une partie non négligeable des candidats accompagnés par le dispositif de branche $(36 \%)$ connaissait des résultats de validation plus défavorables que les moyennes nationales et de manière relativement stable d'une année à l'autre et que la majorité des candidats non accompagnés par ce dispositif (89\%) connaissaient des résultats très défavorables.

\section{Tableau 5}

Typologie des combinaisons régionales de validation au DEES (2005-2006) et résultats du dispositif de branche (DSB)

\begin{tabular}{|c|c|c|c|c|c|c|c|c|}
\hline \multirow[b]{4}{*}{$\begin{array}{l}\text { Candidats } \\
\text { à la VAE }\end{array}$} & \multicolumn{8}{|c|}{ Typologies des tendances de validations régionales (2005-2006) } \\
\hline & \multicolumn{4}{|c|}{2 combinaisons favorables } & \multicolumn{4}{|c|}{2 combinaisons défavorables } \\
\hline & \multicolumn{2}{|c|}{$\begin{array}{l}>\quad[V T>V P+N V] \\
\text { Validations totales }>50 \\
\% \text { et Non-validations } \\
<10 \%\end{array}$} & \multicolumn{2}{|c|}{$\begin{array}{l}>\quad \text { Zéro validation } \\
\text { Non-validation }\end{array}$} & \multicolumn{2}{|c|}{$\begin{array}{l}>\quad[\mathrm{VP}+\mathrm{NV}>\mathrm{VT}] \\
\text { Validations partielles }> \\
37 \% \\
\text { et Non-validations }>10\end{array}$} & \multicolumn{2}{|c|}{$\begin{array}{l}>\quad[N V++] \\
\text { Non-validations }\end{array}$} \\
\hline & Total* & DSB** & Total & DSB & Total & DSB & Total & DSB \\
\hline Nombre & 485 & 400 & 74 & 62 & 3621 & 260 & 700 & 0 \\
\hline$\%$ & 10 & 55 & 2 & 9 & 74 & 36 & 14 & 0 \\
\hline $\begin{array}{l}\text { Total } \\
\text { canditats aux jurys } \\
(4880)\end{array}$ & \multicolumn{4}{|c|}{$559 / 4880$ (11\%) } & \multicolumn{4}{|c|}{$4321 / 4880$ (89\%) } \\
\hline $\begin{array}{l}\text { Dont candidats DSB } \\
\text { (722) }\end{array}$ & \multicolumn{4}{|c|}{$462 / 722(64 \%)$} & \multicolumn{4}{|c|}{$260 / 722(36 \%)$} \\
\hline
\end{tabular}

$T^{*}$ : Total : total des candidats se présentant.

** : DSB : par des candidats passés par le dispositif de soutien de branche.

Note de lecture : Dans la première combinaison favorable, les validations totales (VT) sont plus nombreuses que la somme des validations partielles (VP) et des non-validations (NV). On y dénombre 485 candidats à la VAE (soit $10 \%$ des validations) dont 400 passent par le dispositif de branche (soit $55 \%$ du dispositif).

Les deux combinaisons favorables recouvrent 559 des candidats (soit $11 \%$ ) et 462 candidats passés par le dispositif de branche (soit $64 \%$ du dispositif).

\section{Sigles :}

DEES : diplôme d'État d'éducateur spécialisé.

VT : Validations totales.

VP: Validations partielles.

NV : Non-validations.

Note : les tendances de validation ont été calculées en fonction de leurs écarts aux moyennes nationales, tous candidats confondus.

Source : Rapport de Rozario, 2008. 


\subsection{Une efficacité qui dépend de la sélection des candidats ou de leur accompagnement?}

Ces résultats conduisent les partenaires sociaux à s'interroger sur l'efficacité de la sélection et du parcours d'accompagnement mis en place dans le dispositif de soutien de branche et sur les causes des résultats défavorables obtenus par les candidats suivis, malgré l'investissement mis en place : $9 \%$ ne valident aucune fonction et $36 \%$ ont des résultats plutôt défavorables, malgré le dispositif imaginé. Les résultats positifs sont-ils dus à la sélection ou au parcours d'accompagnement VAE ? D'autres facteurs expliquent-ils les résultats négatifs observés, même s'ils sont significativement moins importants dans le cas des candidats suivis par le dispositif de branche?

Le croisement des données sur les résultats de l'hypothèse de bonne fin (la chance de validation une, deux, trois ou bien quatre fonctions sur quatre du référentiel d'éducateur spécialisé), les résultats de validation des 722 candidats DSB s'étant présenté aux jurys et les quatre types de combinaisons de validation observés conduisent à deux constats :

- les candidats suivis par le dispositif d'accompagnement de branche connaissent des résultats significativement plus favorables que les autres candidats : soit plus de validations totales et significativement moins d'absences de validation pour $64 \%$ des candidats DSB contre seulement $11 \%$ des candidats hors dispositif et seulement $36 \%$ de candidats DSB avec des résultats plutôt défavorables (un taux de validations totales significativement moins important et un taux d'absences de validation plus élevé) contre $89 \%$ des candidats hors dispositif (voir le tableau 5) ;

- les résultats positifs de validation sont davantage dus au parcours d'accompagnement qu'à la sélection des candidats. En effet, très peu d'écoles du travail social ont sélectionné les candidats (9 sur 79). Lorsque ceux-ci étaient sélectionnés, "l'hypothèse de bonne fin » de résultats ne s'est avérée fiable que pour $75 \%$ des candidats à qui l'on avait pronostiqué une validation totale. Elle varie pour tous les autres pronostics et candidats.

Notons également que sur les 79 écoles du travail social impliquées dans le dispositif de soutien de branche à la VAE, très peu ont appliqué l'hypothèse de bonne fin comme processus de sélection initial des candidats dans le parcours prévu :

- neuf écoles l'ont strictement appliquée, dans le sens où les candidats que l'on estimait n’avoir la chance de valider que deux fonctions du référentiel de diplômes sur les quatre n’ont pas été retenus pour entrer dans le dispositif de soutien ;

- onze écoles ont utilisé l'hypothèse de bonne fin soit pour orienter les candidats vers les diplômes correspondant le plus à leur expérience, soit pour les conseiller sur les fonctions qu’ils auraient intérêt à valider compte-tenu de leur expérience ;

- enfin, dix sept écoles n’ont pas utilisé ce processus de sélection en amont du parcours, après avoir estimé qu'il était contraire au droit. 
Peut-on déduire l'existence d'un management régional de la VAE, encadrant finalement l'ensemble des démarches de VAE et des dispositifs associés ? Quelles considérations le motivent et sur quels leviers agit-il ? Les neuf audits régionaux (379 entretiens) et l'interrogation de 81 acteurs plus directement impliqués dans l'organisation régionale de la VAE confirment cette hypothèse. Deux facteurs de gestion sont utilisés pour orienter les résultats de validation en fonction de préférences et de considérations détaillées ci-après : la doctrine de recevabilité administrative des candidats et les consignes de validation transmises aux membres de jury.

\subsection{Le management régional de la VAE par la doctrine de recevabilité des candidatures}

Les résultats de validation d'une session de jury d'une année à l'autre, mais aussi par comparaison avec les résultats des autres régions, constituent des indicateurs sensibles pour les organisateurs de la VAE, en particulier le taux de validations totales et le taux de validation nulles. Un taux élevé comme un faible taux de validation totale inquiètent : dans le premier cas, aurait-on "donné " le diplôme et dans l'autre, "saqué » les candidats par VAE ? Par ailleurs, personne ne sait comment interpréter et gérer les candidats n’obtenant aucune validation, ni ceux qui ne se présentent pas aux jurys. Comment limiter les validations nulles, compte-tenu des coûts élevés et de l'investissement exigé par la procédure de VAE ? Comment éviter les reports de présentation devant les jurys ?

Pour mieux gérer ces incertitudes et ces résultats, se mettent progressivement en place des commissions régionales dites " intervalideurs " ou " intercertificateurs ». Elles associent principalement le rectorat, le Dava (Dispositif académique de validation des acquis) et la Drass (Direction régionale des affaires sanitaires et sociales), et dans une moindre mesure le responsable VAE DSB DEES d'une école du travail social et le conseil régional (via le responsable VAE ou bien le responsable de la cellule d'information conseil en VAE, voir la phase " amont " du schéma 1).

\section{Tableau 6}

Les 4 types d'acteurs régionaux intervenant dans l'organisation de la VAE DEES (diplôme d'État d'éducateur spécialisé)

\begin{tabular}{|c|c|c|c|c|c|c|c|c|c|c|}
\hline \multirow{3}{*}{$\begin{array}{l}\text { Acteurs } \\
\text { Entretien } \\
\text { conduits }\end{array}$} & \multicolumn{10}{|c|}{ Parties prenantes régionales de la VAE (Educateur spécialisé) - Système d'action concret* } \\
\hline & \multicolumn{4}{|c|}{$\begin{array}{l}\text { Rectorat / Académie } \\
\text { (Education nationale) }\end{array}$} & \multirow{2}{*}{\begin{tabular}{|c}
$\begin{array}{c}\text { Drass } \\
\text { (Affaires }\end{array}$ \\
sociales) \\
Inspecteurs
\end{tabular}} & \multicolumn{2}{|c|}{$\begin{array}{l}\text { Ecoles du } \\
\text { travail social }\end{array}$} & \multicolumn{3}{|c|}{$\begin{array}{l}\text { Acteurs } \\
\text { régionaux }\end{array}$} \\
\hline & Dava & $\begin{array}{l}\text { Services } \\
\text { des } \\
\text { examens }\end{array}$ & $\begin{array}{c}\text { Accompa- } \\
\text { gnateurs } \\
\text { VAE }\end{array}$ & Inspecteurs & & $\begin{array}{l}\text { Conseillers } \\
\text { techniques }\end{array}$ & $\begin{array}{l}\text { Respon- } \\
\text { sables VAE }\end{array}$ & $\begin{array}{c}\text { Respon- } \\
\text { sables } \\
\text { VAE }\end{array}$ & $\begin{array}{l}\text { CRIS/ } \\
\text { Carif } \\
\text { Oref }\end{array}$ & Autres \\
\hline \multirow[t]{2}{*}{81} & 11 & 7 & 6 & 2 & 10 & 4 & 26 & 8 & 6 & 1 \\
\hline & \multicolumn{4}{|c|}{$26(32 \%)$} & \multicolumn{2}{|c|}{$14(17 \%)$} & $32 \%$ & \multicolumn{3}{|c|}{$15(19 \%)$} \\
\hline
\end{tabular}


Note de lecture : * Au sens de l'analyse stratégique (Sainsaulieu, 1988), c'est-à-dire représentatif du management régional de la VAE pour ce diplôme.

Sigles :

Carif Oref: Centre de ressources et d'information sur la formation et l'emploi.

Cons. Tech. : Conseillers techniques (Drass).

Cris : Cellules régionales interservices (chargées de l'information VAE entre autres).

Dava : Dispositif académique de validation des acquis.

Drass : Direction régionale des affaires sanitaires et sociales.

Source : Rapport de Rozario, 2008.

Ces commissions examinent les résultats de validation et optent pour une doctrine régionale de recevabilité des demandes de VAE (livret 1) soit " ouverte » (Alsace, Bourgogne, Centre, Ile-de-France, Rhône-Alpes), soit " sélective " (Aquitaine, Bretagne, MidiPyrénées, Picardie). L'objectif de cette doctrine est d'intervenir en amont sur la sélection des candidatures VAE. Dans certaines régions, les options prises se réalisent sans commission dédiée, entre acteurs ou bien par l'acteur qui contrôle la procédure de recevabilité. Notons qu'au moment de l'évaluation, cet acteur n'est pas nécessairement le rectorat via le service des examens où équivalent. Par surcharge de travail, manque de moyen ou par choix de délégation, il peut s'agir du Dava, de la Drass ou bien d'une école du travail social.

- La recevabilité administrative « ouverte " comprend essentiellement des activités de contrôle : vérification du cadre de la demande (une seule demande pour le même diplôme dans la même académie et trois demandes maximum de VAE) ; présence des informations demandées et vérification de leur authenticité (signatures, dates...) ; vérification et calcul de la durée des expériences (trois ans obligatoirement dans le champ du diplôme), en éliminant les périodes de formation initiale et continue ainsi que les stages professionnels non éligibles pour la VAE.

Il est intéressant de noter que les acteurs adoptant cette doctrine la justifient par une certaine conception de la VAE : tout candidat peut faire la preuve des acquis de son expérience, même si les premières informations administratives ne sont pas totalement probantes. Le processus d'auto-évaluation suivant la recevabilité leur permettra peut-être d'en faire la preuve, ou pas. Dans tous les cas, les jurys sont souverains et seuls juges d'une candidature. Certains acteurs adoptent cette doctrine de recevabilité par conviction ; d'autres lorsque les résultats de validation sont jugés défavorables aux candidats ou bien lorsqu'un besoin d'éducateurs spécialisés diplômés se fait sentir au niveau régional.

- La recevabilité administrative " sélective " ajoute au contrôle administratif précédemment décrit d'autres critères : l'ajout d'une lettre de motivation, des tableaux de description plus détaillés des expériences ou bien plus de pages de description que prévu et parfois même, la transmission du référentiel d'éducateur spécialisé. D’autres critères peuvent intervenir : la sélection des diplômés de l'éducation spécialisé uniquement, de niveau de diplôme le plus proche et inférieur au niveau III, ceux qui présentent plus de 
quatre expériences significatives ou encore un parcours auprès de publics et d'établissements différents.

Les acteurs interviewés justifient cette doctrine par une consigne figurant dans les circulaires d'application de la VAE DEES : "vérifier que l'expérience est en rapport avec le diplôme " et la considération sur la " dangerosité " potentielle de certains candidats VAE, compte-tenu de la particularité des publics de l'éducation spécialisée (personnes âgées, enfants, mineurs sous tutelle, familles en difficulté, handicapés...).

Derrière cette doctrine en faveur de la sélection des candidats estimés les plus proches de la validation, se profile également une conception de la VAE différente de la première. Les candidats éligibles sont ceux qui ont déjà des acquis d'expérience probants et quasi des éducateurs spécialisés. Dans ce cas, l'accompagnement est jugé peu utile car dès le livret 1 , l'écrit du candidat est convainquant. Comme dans le cas précédent, certains acteurs adoptent cette doctrine par conviction ; d'autres, pour réduire les coûts de la VAE, les abandons, les reports de présentation du livret 2 devant le jury et la complexité d'organisation des parcours complémentaires en cas de validations partielles.

\subsection{Le pilotage régional par la professionnalisation des membres de jury}

Lorsque surviennent des configurations de validation jugées défavorables, l'ensemble des acteurs interviewés incrimine les membres de jury et les 64 membres de jury interviewés se plaignent quant à eux des conditions très difficiles d'organisation et de la complexité d'évaluation des candidatures du diplôme d'État d'éducateur spécialisé. S’ajoute alors au souci de piloter les résultats de validation en fonction de préférences régionales, la question de la "professionnalisation des membres de jury ", deuxième levier d'action du pilotage régional de la VAE.

Tableau 7

Typologie représentative des membres de jury interviewés sur les dilemmes de validation dans neuf régions

\begin{tabular}{|c|c|c|c|c|c|c|c|c|}
\hline \multirow{3}{*}{$\begin{array}{l}\text { Collèges } \\
\text { Entretiens } \\
\text { conduits }\end{array}$} & \multicolumn{8}{|c|}{ Parties prenantes des Jurys de VAE (Educateur spécialisé) - Système d'action concret* } \\
\hline & \multicolumn{2}{|c|}{ Vice-présidence Drass } & \multicolumn{2}{|c|}{ Professionnels } & \multirow{2}{*}{$\begin{array}{l}\text { Formateurs } \\
\text { Ecoles du travail } \\
\text { social }\end{array}$} & \multicolumn{3}{|c|}{ Administrations publiques } \\
\hline & Inspecteurs & $\begin{array}{l}\text { Conseillers } \\
\text { techniques }\end{array}$ & Employeurs & Salariés & & $\begin{array}{l}\text { Education } \\
\text { nationale } \\
\text { (Dava) }\end{array}$ & $\begin{array}{l}\text { Justice } \\
\text { (PJJ) }\end{array}$ & $\begin{array}{l}\text { Jeunesse } \\
\text { et Sport }\end{array}$ \\
\hline \multirow[t]{2}{*}{64} & 9 & 4 & 22 & 1 & 14 & 9 & 3 & 2 \\
\hline & $14 \%$ & $6 \%$ & $34 \%$ & $2 \%$ & $22 \%$ & $14 \%$ & $5 \%$ & $3 \%$ \\
\hline
\end{tabular}

* : Au sens de l'analyse stratégique (Sainsaulieu, 1988), c'est-à-dire representatif de la composition de jurys VAE pour ce diplôme. Sigles : Drass : Direction régionale des affaires sanitaires et sociales. FI : Formation initiale. Dava : Dispositif académique de validation des acquis. PJJ : Protection judiciaire de la jeunesse. DEES : Diplôme d'Etat d'éducateur spécialisé. Source : Rapport de Rozario, 2008. 
Les membres de jury interviewés invoquent deux principales difficultés de validation pour ce diplôme :

- La dimension pluri-acteurs de ce diplôme, décrite en introduction, convoque des cultures administratives et des cultures professionnelles jugées différentes, voire opposées dans leurs conceptions de la validation des acquis de l'expérience. Les conflits entre membres de jury font partie des fréquents dilemmes de validation décrits. Le tableau 8 illustre cette variété d'appartenance et de références au travers des différentes catégories de jury (les " collèges ");

- La nouveauté du référentiel d'éducateur spécialisé diffusé par l'arrêté du 12 mars 2004. Si les fonctions 1, 2 et 3 décrivent la chronologie des activités professionnelles attendues passant par la construction d'une relation interindividuelle dans un contexte de commande sociale (fonction 1), l'accompagnement éducatif d'une personne ou d'un groupe (fonction 2) et le développement/l'éducation de la personne ou d'un groupe par le choix d'actions spécifiques (fonction 3), la fonction 4 relève d'un autre registre. Elle insiste sur la capacité de l'éducateur spécialisé à entretenir sa professionnalité, à l'instar du modèle du praticien réflexif ${ }^{5}$ de Donald Schön (1983), référence explicite des concepteurs du référentiel. L'évaluation des candidatures au diplôme apparaît complexe.

Dans ce contexte, les acteurs s'accordent sur la nécessité de professionnaliser les membres de jury et d'améliorer la qualité de l'organisation et du management des jurys. Les organisateurs (principalement les Drass et les services du rectorat) interviennent sur les consignes de validation transmises au moment des sessions d'information et d'actions de formation spécifiques (dans ce cas, les organismes de formation sont impliqués). Organisateurs ou formateurs produisent également des documents précisant les critères d'évaluation des livrets 2. Comprendre les résultats de VAE implique que l'on s'intéresse donc également aux consignes de validation transmises aux membres de jury, variables selon les documents, les régions et les cultures de reconnaissance de l'expérience en présence, comme le montrent l'analyse de plus de 100 dilemmes de validation rencontrés par une soixantaine de membres de jury.

\subsection{Les dilemmes de jury révèlent trois cultures de la reconnaissance}

L'analyse croisée des dispositifs de professionnalisation mis en place dans les neuf régions d'enquête, des dilemmes de validation rencontrés par les 64 membres de jury et de la réception des candidats (91 salariés suivis par le dispositif de soutien de branche

\footnotetext{
5. D. Schön a défendu l'idée que le praticien (celui qui pratique) était capable de concevoir une théorie de sa pratique susceptible de la faire évoluer contre l'idée que seuls les professionnels diplômés et les experts étaient capables de ce type de réflexivité. Son modèle a largement inspiré les tenants de l'analyse de pratique et les promoteurs de l'expérience comme source de savoirs et d'expertise.
} 
échantillonné sur tous les profils de validation) indique l'existence non pas d'une mais de trois formes légitimes de validation. Celles-ci se distinguent, comme nous allons le voir, par des conceptions différentes, voire conflictuelles de ce que doit être et ne pas être la bonne candidature à la VAE et en conséquence, sur ce qui fait le plus sens dans l'évaluation et la validation.

a) Le jury « docimologique » s'attache à contrôler la conformité de la candidature décrite dans le livret 2, en considérant que l'essentiel y est consigné. Présence des informations demandées, cohérence, adéquation (entre ces informations et le référentiel du diplôme) et conformité en sont les principaux critères. Il convient souvent aux membres de jury peu familiers de l'éducation spécialisée et/ou soucieux d'adopter une méthode quantifiée, dont ils peuvent discuter au moment des délibérations. L'enjeu consiste à se forger un système d'évaluation par pondérations, pourcentages ou notes dont le cumul approchera la validation la plus précise et juste possible. Ce modèle prête peu attention à l'originalité du livret 2, à la motivation du candidat, à l'exemplarité et à l'originalité de son parcours. Durant l'entretien, le jury docimologique prépare des questions de vérification pour combler les zones incomplètes ou incohérentes du livret 2, laissant souvent aux candidats l'impression qu'il ne s'intéresse qu'à la collection de leurs manques. S'il est satisfait du livret 2, il lui arrive de ne poser aucune question lors de l'entretien, comportement interprété comme un manque de travail ou d'intérêt pour la candidature.

b) Le jury " promotionnel » évalue les qualités d'un professionnel de l'éducation spécialisée reconnu pour son parcours, sa motivation et sa personnalité. Dans ce cas, le livret 2 est considéré comme une preuve suffisamment probante de la capacité à identifier des acquis d'expérience en lien avec le diplôme. Il sert plutôt de base à la vérification de l'authenticité des expériences et analyses décrites ainsi qu’au repérage de comportements ou d'analyses potentiellement révélateurs de l'éthique du candidat, c'est-à-dire de sa manière d'appliquer en situation les valeurs de l'éducation spécialisée. Eviter la "dangerosité " potentielle de certains candidats motive ce type de consigne de validation, d'ailleurs suscitée par les circulaires d'application de la VAE DEES. Les candidats sont questionnés sur des cas critiques d'intervention et sur la fonction 4 du référentiel d'éducateur spécialisé "Construction d'un cadre d'analyse et d'une expertise des pratiques éducatives et sociales", jugée caractéristique de la responsabilité de l'éducateur spécialisé. Sommés de justifier les raisons de leurs choix d'actions et d'analyses, les candidats se sentent souvent remis en cause professionnellement.

c) Le jury " transversal » vérifie principalement la transférabilité et la solidité des acquis d'expérience présentés dans le livret 2 , en considérant que le diplôme représente une qualification générique dépassant les contextes opératoires de la compétence. La transférabilité des acquis se vérifie par le nombre d'établissements fréquentés et la variété des publics traités. Ce type de jury utilise autrement la fonction 4 du référentiel d'éducateur spécialisé : prisme de lecture, elle sert à apprécier l'ensemble du livret 2 à l'aune d'une capacité du candidat à décontextualiser ses apprentissages les plus probants. L'entretien se prête à 
la vérification de la solidité et du degré d'approfondissement des savoirs professionnels acquis par l'expérience : les cas décrits pour les fonctions 1,2 et 3 du référentiel sont interrogés avec le critère réflexif de la fonction 4 . Les candidats perçoivent le jury comme décalé dans ses questions, qui sont éloignées du travail fourni dans le livret 2 qu'ils s’apprêtent à défendre oralement.

Ces préférences de validation génèrent des attentes normatives sur les éléments de candidature, à savoir le dossier écrit présenté aux jurys (« le livret 2 » dans le vocabulaire du ministère de l'Education nationale) et l'entretien, mais aussi l'accompagnement, synthétisées dans le tableau 8. En cas d'accompagnement, les jurys jugent plus sévèrement ces éléments de candidature s'ils ne sont pas conformes à leurs attentes. Ces attentes normatives permettent aux membres de jury de distinguer la candidature adéquate de celle qui ne l'est pas au niveau du dossier de preuves d'acquis d'expérience (le livret 2) et au niveau de l'entretien avec le candidat. Lorsque le dossier écrit et les interactions entre le candidat et le jury ne correspondent pas à ces attentes, les candidatures sont jugées inadéquates, que le candidat ait été accompagné ou pas et quelle que soit la qualité de cet accompagnement. Souvent ignorées des accompagnateurs, des candidats et des membres de jury eux-mêmes, ces attentes normatives engendrent, du côté des candidats interrogés, soit l'impression d'une entente entre acteurs du jury lorsqu'elles sont partagées, soit des incompréhensions et des discordes parfois violentes entre eux lorsqu'elles divergent.

\section{Tableau 8}

Les attentes normatives des trois modèles de validation des jurys VAE sur les éléments de candidature au DEES (diplôme d'État d'éducateur spécialisé)

\begin{tabular}{|l|l|l|l|}
\hline & $\begin{array}{c}\text { DAttentes par rapport } \\
\text { à la pr ésentation et au } \\
\text { contenu du Livret 2 } \\
\text { (dossier remis aux jurys } \\
\text { pour évaluation) }\end{array}$ & $\begin{array}{c}\text { Attentes par } \\
\text { rapport au candidat } \\
\text { au moment de } \\
\text { l'entretien }\end{array}$ & $\begin{array}{c}\text { Attentes } \\
\text { par rapport à } \\
\text { l'accompagnement }\end{array}$ \\
\hline $\begin{array}{l}\text { Jury } \\
\text { "Docimologique » } \\
\begin{array}{l}\text { Un exercice } \\
\text { d'évaluation réussi } \\
\text { et conforme }\end{array}\end{array}$ & $\begin{array}{l}\text { II doit être complet, conforme à la } \\
\text { structure initiale sans changement } \\
\text { d'intitulé ni de place des rubriques } \\
\text { et détaillé, fonction par fonction, } \\
\text { pour toutes les rubriques. }\end{array}$ & $\begin{array}{l}\text { La maîtrise des informations } \\
\text { du livret 2 et la préparation } \\
\text { de réponses satisfaisantes } \\
\text { aux questions sur les points } \\
\text { faibles et aux manques } \\
\text { constatés de l'écrit. }\end{array}$ & $\begin{array}{l}\text { L'appui à la production } \\
\text { d'un livret 2 conforme, } \\
\text { complet et détaillé et } \\
\text { la préparation orale des } \\
\text { candidats à argumenter } \\
\text { sur les points faibles } \\
\text { du livret 2. L'aide à } \\
\text { la mise en forme du } \\
\text { livret 2 (sommaire, } \\
\text { pagination, correction } \\
\text { des fautes de français, de } \\
\text { l'expression...). }\end{array}$ \\
\hline
\end{tabular}




\begin{tabular}{|c|c|c|c|}
\hline $\begin{array}{l}\text { Jury } \\
\text { « Promotionnel » } \\
\text { Un parcours original et } \\
\text { un praticien motivé et } \\
\text { responsable }\end{array}$ & $\begin{array}{l}\text { II doit exprimer la personnalité } \\
\text { et les points de vue du candidat, } \\
\text { comprendre des informations } \\
\text { détaillées et des analyses indiquant } \\
\text { la posture du candidat par rapport } \\
\text { à l'éducation spécialisée et au } \\
\text { métier. } \\
\text { II doit être original et unique. } \\
\text { Les parties du livret } 2 \text { portant } \\
\text { sur la motivation, le parcours } \\
\text { et les formations suivies sont } \\
\text { particulièrement analysées. }\end{array}$ & $\begin{array}{l}\text { Une motivation forte } \\
\text { par rapport au métier } \\
\text { d'éducateur spécialisé et } \\
\text { une implication personnelle } \\
\text { dans ce métier. La capacité à } \\
\text { débattre et discuter avec les } \\
\text { membres de jury sur la vision } \\
\text { du métier et ses limites. }\end{array}$ & $\begin{array}{l}\text { L'appui à la production } \\
\text { d'un livret } 2 \text { personnel, } \\
\text { original, mettant en } \\
\text { valeur la personne et le } \\
\text { métier. La préparation à } \\
\text { un entretien portant sur } \\
\text { la posture d'éducateur } \\
\text { spécialisé et la motivation } \\
\text { à confirmer sa vocation } \\
\text { pour le métier représenté } \\
\text { par le diplôme. }\end{array}$ \\
\hline $\begin{array}{l}\text { Jury } \\
\text { «Transversal » } \\
\text { Une qualification } \\
\text { collective à partir d'acquis } \\
\text { singuliers }\end{array}$ & $\begin{array}{l}\text { Un livret } 2 \text { démontrant la } \\
\text { transversalité des acquis } \\
\text { d'expériences: des expériences } \\
\text { variées, des publics différents, des } \\
\text { liens entre les expériences décrites } \\
\text { dans chacune des fonctions ou } \\
\text { bien une seule expérience riche } \\
\text { illustrant les quatre fonctions. Un } \\
\text { parcours de formations et de stage } \\
\text { riches indiquant des intérêts pour } \\
\text { s'auto former au métier et faire } \\
\text { évoluer ses pratiques. }\end{array}$ & $\begin{array}{l}\text { La capacité à prendre du } \\
\text { champ par rapport aux } \\
\text { descriptions contextualisées } \\
\text { du livret } 2 \text {; à imaginer la } \\
\text { transposition de ses acquis } \\
\text { d'expériences dans d'autres } \\
\text { environnements et à utiliser } \\
\text { la fonction } 4 \text { du métier vu } \\
\text { par le diplôme « Construction } \\
\text { d'un cadre d'analyse et d'une } \\
\text { expertise des pratiques } \\
\text { éducatives et sociales », pour } \\
\text { évaluer de manière critique } \\
\text { chaque expérience. }\end{array}$ & $\begin{array}{l}\text { L'appui au choix } \\
\text { d'expériences dont les } \\
\text { acquis peuvent être } \\
\text { transposables dans } \\
\text { d'autres environnements. } \\
\text { L'adoption des } \\
\text { compétences décrites } \\
\text { dans la fonction } 4 \text { et } \\
\text { leur utilisation à l'oral } \\
\text { (auto-évaluation, analyse } \\
\text { critique de sa pratique, } \\
\text { recherche d'amélioration } \\
\text { de ses ressources dans le } \\
\text { temps, etc. ). }\end{array}$ \\
\hline
\end{tabular}

Source : Rapport de Rozario, 2008.

Ainsi, les jurys "docimologiques " n'admettent pas des livrets 2 incomplets, ne respectant pas les consignes à la lettre ou bien mal présentés. Les jurys " promotionnels " ne comprennent pas l'accompagnement uniquement centré sur la technique de rédaction du livret 2 et l'exercice de VAE, car il s'agit d'accéder à une communauté professionnelle ayant d'autres exigences. Les jurys «transversaux », enfin, jugent sévèrement l'accompagnement aboutissant à ne formaliser que des acquis de compétences, peut-être probants mais trop contextualisés. La VAE ne fait pas que reconnaître un très bon professionnel, elle le diplôme. S'agit-il de reconnaître la qualité d'un professionnel dont les compétences sont déjà avérées au regard d'un référentiel et décrites de manière conforme ? Reconnaître le parcours et le potentiel d'un professionnel intéressant une communauté professionnelle ? Reconnaître la qualité d'un professionnel capable - car le référentiel de certification du diplôme d'État d'éducateur spécialisé est ainsi construit -, d'auto-analyser et d'évaluer sa pratique de manière réflexive?

Ces interrogations préalables à la décision se posent aussi bien dans le cadre d'une démarche individuelle que dans celui d'une démarche collective, puisque la VAE est un droit individuel qui participe aux politiques de gestion de la main-d'œuvre, en figurant 
au titre II « Travail, emploi et formation professionnelle » de la loi 2002-73 de modernisation sociale du 17/01/2002.

\section{Conclusion : La VAE, un droit plurivoque à décrypter}

Le lecteur aura compris l'importance des facteurs d'organisation et de gestion de la VAE, intrinsèquement liés à la gouvernance nationale des diplômes d'État, dont le secteur du travail social est une illustration marquante. L'analyse du pilotage régional de la VAE a également permis de décrire des stratégies d'acteurs rarement dépeintes dans les études de la VAE mais aussi les dimensions fondamentalement culturelles des mandats de reconnaissance attribués à la VAE. Que ce soit à titre individuel ou dans le cadre d'un dispositif d'entreprise (sectoriel/collectif), les acteurs de la VAE et ses analystes ne peuvent ignorer ces facteurs stratégiques et symboliques, déterminants dans les manières de faire VAE. Ces résultats semblent utiles dans un contexte où les observations portent majoritairement sur des dimensions individuelles et pédagogiques de la VAE - les motivations des candidats, l'accompagnement et ses "métiers », la mobilité professionnelle exprimée par un changement de salaire et de catégorie socio-professionnelle et le vécu des candidats - tant l'idéologie méritocratique et individuelle de ce droit est forte. C'est notamment ce que rappelle le rapport français remis au Cedefop (Centre européen de développement de la formation professionnelle) sur la " Gouvernance de la formation professionnelle ", qui s'appuie sur l'analyse comparée des travaux produits sur la VAE depuis les années 2000 (de Rozario, 2009). Plusieurs autres travaux soulignent également la nécessité d'ouvrir les cadres d'analyse de la VAE (Neyrat, 2007 ; Teissier et Rose, 2006 ; Dupray et al, 2003 notamment) Les initiatives et pratiques mises en place sous couvert de "professionnalisation » méritent donc d'être mieux prises en considération dans la mesure où elles influent fortement la sélection des candidats, le processus, mais aussi les jugements de validation. Nous gagnerions à énoncer clairement ces conceptions et leurs divergences pour en discuter, perspective assez difficile dans un domaine qui touche au point sensible de la reconnaissance des personnes par l'État, via les diplômes.

Face aux politiques et aux façons dont la VAE est reçue, nous sommes donc invités à élargir et enrichir les indicateurs d'évaluation de ce droit (les acteurs restent trop focalisés sur les résultats moyens de validation sans prendre en considération les combinaisons régionales de validation et leurs tendances dans le temps) et les cadres d'analyse usuels de la VAE. Il ne s'agit pas que d'une pratique d'accompagnement pédagogique de type herméneutique où l'angle d'analyse est l'interaction entre le candidat et l'accompagnateur ou d'éventuels nouveaux métiers associés à ce type de pratique (vision pédagogique et psychologique de la VAE, voir Prot, 2003). On ne peut la réduire non plus à une stricte politique de mobilité individuelle avec des indicateurs statistiques classiques et l'intention de montrer en quoi la VAE comme la formation compense les inégalités et infère les trajectoires (perspective sociologique des politiques publiques, Paul et Rose, 2008). Les résultats présentés plaident en faveur d'une analyse plus critique de l'exercice du droit au 
niveau de la gouvernance et du management de la VAE car les pratiques véhiculent des conceptions distinctes de ce qu'il est légitime ou non de reconnaître d'une expérience, qu'il conviendrait de mieux décrypter (Pinte, 2002, Tallard, Théret et Uri ; 2000, Tanguy et al., 1986). Touchant la question de la reconnaissance (Honneth, 2004), la VAE mérite une lecteur anthropologique et symbolique rarement mise en exergue, perspective qui nous a notamment permis de mieux comprendre les attentes normatives à partir desquelles délibèrent ceux qui jugent la valeur d'une expérience par rapport aux exigences d'un diplôme national dont se porte garant l'État. En la matière, il faut souligner l'encadrement européen des politiques nationales de mobilité et de reconnaissance et l'intérêt de les considérer "à distance ", sinon " avec distance ", par comparaisons, afin de mieux repérer les cultures de la reconnaissance à l'œuvre.

\section{Bibliographie}

Caillot L. (2007), La validation des acquis de l'expérience en vue de l'obtention des diplômes délivrés par les ministères chargés des affaires sociales et de la santé : réalisations 2006 et prévisions 2007, Paris : DGAS, $52 \mathrm{p}$.

Capul, M. Lemay M. (2007), De l'éducation spécialisée ? Paris : Erès, 448 p.

CEE, Bureau M.C. et al. (2008), Politique des entreprises en matière de certification et l'utilisation de la VAE. Paris : Rapport de recherche pour la Dares, $\mathrm{n}^{\circ}$ 46, $227 \mathrm{p}$.

Chanlat J.-F. (2007), L'individu dans l'organisation, les dimensions oubliées, Laval : Presses de l'université de Laval, Eksa, 842 p.

Chardon O., Estrade M.-A., (2007), Les métiers en 2015, Rapport. Paris : Direction de l'animation et de la recherche des études et des statistiques et Centre d'analyse stratégique, Coll. «Qualifications \& prospective », 179 p.

Chopart J.-N. (dir.) (2000), Les mutations du travail social, dynamiques d'un champ professionnel. Paris : Dunod, DRESS, 303 p.

Conseil supérieur du travail social (2007), Décloisonnement et articulation du sanitaire et du social. Rennes : ENSP, coll. « Rapports du CSTS », 120 p.

Credoc, Lerfas et Grefoss (2002), Contrat d'etudes prospectives social et médico-social de la branche professionnelle des établissements à but non lucratif. Paris : Credoc, 374 p.

De Rozario P. (dir.) (2005), VAP et entreprises, enjeux politiques et systèmes de reconnaissance des apprentissages, Tome 3, Huit monographies sectorielles. Paris : Cnam, $374 \mathrm{p}$.

De Rozario P. (dir.) (2008), Evaluation du DSB VAE DEES (dispositif de soutien de branche à la VAE pour le Diplôme d'Etat d'éducateur spécialisé), Rapport final. Paris : Cnam (Umr Cnrs LISE), 338 p. 
De Rozario P. (2010), "Governance of Vet Systems", Topic one, in National Vet research report : France 2009, Centre Inffo, Réseau ReferNet, Thessaloniki, Cedefop, October 2009, pp. 7-12.

De Rozario P. (2012), "Culture et gestion de la vae dans le travail social ", in La reconnaissance et la validation des acquis de l'expérience, Gilles Pinte, Carriérologie, $\mathrm{n}^{\circ} 12$, Montréal : Université du Québec à Montréal , pp. 440-461.

Dubouchet L., Berlioz G. (2005), Evaluation du dispositif d'information-conseil en VAE. Paris : ministère de l'Emploi, du travail et de la cohésion sociale, $73 \mathrm{p}$

Dupray A., Guitton C., Montchatre S. (2003), Réfléchir la compétence, approches sociologiques, juridiques, économiques d'une pratique gestionnaire. Octares : Toulouse, 272 p.

Honneth A. (2004), "Visibilité et invisibilité : sur l'épistémologie de la reconnaissance ", in Revue du Mauss "De la reconnaissance. Don, identité et estime de soi ", n²3, pp. 126-150.

Jaeger M. (2006), L'articulation du sanitaire et du social. Travail social et psychiatrie. Dunod,

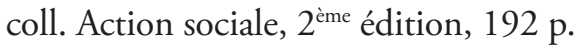

Janvier J.-R. (2004), Mettre en ouvre le droit des usagers dans les organisations sociales et médico-sociales. Paris: Dunod, $3^{\text {ème }}$ édition, $344 \mathrm{p}$.

Observatoire de la branche sanitaire, sociale et médico-sociale à but non lucratif (2007), Enquête Emploi 2007, Enseignements et perspectives. Paris : Unifaf, 127 p.

Le Gales P. (1994), "Du gouvernement des villes à la gouvernance urbaine ", in Revue française de sciences politiques, vol. 45, pp. 57-95.

Maillard F. (2008), Des diplômes aux certifications professionnelles, nouvelles normes et nouveaux enjeux. Rennes: Presses Universitaires de Rennes, 342 p.

Ministères chargés de l'Emploi et de la santé, (2007), Orientation pour les formations sociales 2007-2009. Paris : Direction générale de l'action sociale (DGAS), Dircom 07 056, $53 \mathrm{p}$.

Neyrat F. (dir.) (2007), La validation des acquis de l'expérience, la reconnaissance d'un nouveau droit. Rennes : PUR, $342 \mathrm{p}$.

Paul J.-J., Rose J. (2008), Les relations emploi-formation en 55 questions, Paris : Dunod, $350 \mathrm{p}$.

Pinaud M. (2004), Le recrutement, la formation et la professionnalisation des salariés du secteur sanitaire et social, Rapport Conseil économique et social, $234 \mathrm{p}$.

Pinte G. (2002), « La vae comme point de rencontre entre deux conceptions de la formation des adultes qui se sont opposées à travers l'histoire : la formation professionnelle continue et l'éducation permanente ", in Education permanente, n 150, pp. 95-106 
Prot B. (2003), «Analyse du travail des jurys en validation des acquis : l'usage des référentiels ", in L'orientation scolaire et professionnelle, vol. 32-2, pp. 219-243.

Sainsaulieu R. (1988), L’identité au travail. Paris : Presses de Sciences po., 480 p.

Salmon A. (2010), Mais que font les éducateurs? Le travail social à l'épreuve du politique, Paris : Desclée de Brouwer, 144 p.

Schön D. A. (1983), The Reflective Practitioner: How professionals think in action. London: Temple Smith.

Schön D. A. (1994), Le praticien réflexif : à la recherche du savoir caché dans l'agir professionnel. Paris : Ed. Logiques, 418 p.

Tallard M., Théret B., Uri D. (2000), Innovations institutionnelles et territoires. Paris, l'Harmattan, coll. "Logiques sociales », 449 p.

Tanguy L. (1986), L'introuvable relation emploi-formation, un état des recherches en France. Paris : La Documentation française, $302 \mathrm{p}$.

Teissier J. et Rose J. (2006), «La certification, nouvel instrument de relation emploi-formation, un enjeu français et européen ", Marseille : Céreq-Relief, n 16, 130 p. 